\title{
Optimismo epistémico: en defensa de la objetividad en la ciencia
}

\section{Epistemic optimism: in defense of objectivity in science}

\author{
Roberto Bueno-Cuadra ${ }^{\mathrm{a}, *}$, Elizabeth Dany Araujo-Robles ${ }^{\mathrm{b}}$ \\ aUniversidad de San Martín de Porres, Perú \\ bUniversidad Peruana Cayetano Heredia, Perú
}

\section{Resumen}

El propósito de este trabajo es realizar una defensa de la posición objetivista frente a la insistente presencia de epistemologías constructivistas y relativistas en el campo de la psicología. Para cumplir este objetivo, examinamos críticamente algunos argumentos constructivistas y luego revisamos el papel de los factores no cognoscitivos y del marco conceptual del investigador en la construcción del conocimiento. Finalizamos con una breve discusión de la racionalidad en la decisión científica y de la posibilidad de un desarrollo progresivo, y no solo sustitutivo, del conocimiento científico. Nuestra conclusión es que, contrario a lo que sostienen constructivistas y relativistas, es posible generar conocimiento y que existe una línea real de progreso en el saber humano; a todo lo cual denominamos optimismo epistémico.

Palabras clave: conocimiento científico, constructivismo, objetividad, paradigmas, racionalidad científica, relativismo.

\section{Para citar este artículo:}

Bueno-Cuadra, R., \& Araujo-Robles, E. D. (2017). Optimismo epistémico: en defensa de la objetividad en la ciencia. Liberabit, 23(2), 273-290. doi: 10.24265/liberabit.2017.v23n2.08

\begin{abstract}
The purpose of this paper is to carry out a defense of the objectivist stance before the insistent presence of constructivist and relativist epistemologies in psychology. For this, we critically examine some constructivist arguments and then we review the role of non-cognitive factors and researchers' conceptual frameworks in constructing knowledge. We finalize with a brief account about rationality of scientific decision and the possibility of a progressive, not only substitutive, development of scientific knowledge. We conclude that, contrary to claims of constructivists and relativists, it is indeed possible to create knowledge and there exists a way of true progress in it, all of which we call epistemic optimism.
\end{abstract}

Keywords: scientific knowledge, constructivism, objectivity, paradigms, scientific rationality, relativism.

Este es un artículo Open Access bajo la licencia Creative Commons Atribución-NoComercial-CompartirIgual 4.0

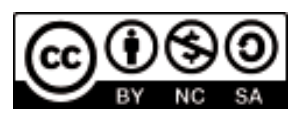


Nuestra visión de la objetividad científica se resume en dos postulados: 1 . El postulado ontológico de que existe un mundo objetivo; es decir, un mundo constituido por entidades definidas y pre-existentes a, e independientes de, cualquier observador (realismo u objetivismo ontológico) y 2. El postulado epistemológico de que es posible llegar a un conocimiento verdadero de ese mundo, en el sentido de que es posible construir acerca de él una imagen que, de acuerdo con la mejor evidencia disponible, se puede juzgar como «correcta» (objetivismo epistemológico). La objetividad epistémica es la posibilidad de evaluar el valor de verdad de un enunciado sobre la base de evidencias y argumentos. Con una limitación: el que un enunciado se estime como verdadero sobre la base de tales evidencias y argumentos no significa que se haya conseguido certeza total. Estos postulados son parte de lo que Searle (1993) denomina la Tradición Racionalista Occidental y Brown (2001) identifica como Ortodoxia Científica. Para Brown, Searle y otros, ambos postulados constituyen el fundamento de la ciencia como la conocemos.

Pero los post-modernos y subjetivistas rechazan los dos postulados. Ellos asumen una ontología constructivista y una epistemología relativista. Para ellos, por consiguiente, cuando se identifica un hecho no se está descubriendo algo pre-existente, independiente de un observador y esto, a su vez, significa que el conocimiento acerca de tal hecho es enteramente relativo a las condiciones de dicho observador. Pero no sólo eso, para el relativista no existe medio alguno que permita decidir entre visiones alternativas acerca del mismo asunto (Baghramian, 2004).

El objetivo de este trabajo es analizar críticamente algunos de los argumentos constructivistas y relativistas. Desde nuestra perspectiva de educadores e investigadores en el campo de la psicología, el tratamiento de estas cuestiones en el seno de nuestra disciplina es más que imprescindible. Sólo pensemos en la posibilidad de que los hechos que son investigados por la psicología y las ciencias sociales en su conjunto no sean más que puras construcciones relativas al observador y que existan tantas «realidades» psicológicas y sociales como investigadores haya. Pero queremos aclarar nuestra posición. Podemos admitir el carácter convencional, socialmente contextualizado y no natural ni universal de muchos de los eventos considerados objetos de estudio de la psicología. Es decir, podemos concordar en que la existencia de probablemente todos esos fenómenos es relativa a un contexto cultural determinado, dado que los hechos psicológicos y en general, los de las ciencias sociales, están constituidos por las prácticas de seres humanos que viven en sociedad. Gergen (1985) y Hacking (1995) son algunos de los muchos autores que han llamado la atención acerca de esto. Así pues, es posible aceptar que muchos de los llamados «fenómenos psicológicos» no existen fuera de un ambiente cultural específico. Más aún, y como ha sido puesto de relieve por Hacking (1995), los conceptos psicológicos exhiben lo que él denomina looping effects, es decir, la capacidad de afectarse a sí mismos (por ejemplo, debido al impacto de las categorizaciones psicológicas sobre los individuos así categorizados). Tal vez es innecesario agregar que tales efectos son enteramente objetivos.

Sin embargo, en el mismo artículo, Gergen (1985) va más allá del carácter convencional de los hechos psicológicos para plantear lo que constituye nuestra preocupación en este trabajo: el cuestionamiento a la objetividad de la propia investigación científica; por ejemplo, cuando hace eco de las conclusiones de ciertos sociólogos del conocimiento científico:

El grado al que una forma dada de comprensión prevalece o es sostenida en el tiempo no depende fundamentalmente de la validez empírica de la perspectiva en cuestión, sino de las vicisitudes de los procesos sociales (e. g., comunicación, negociación, conflicto y retórica). (Gergen, 1985, p. 268) 
En realidad, ya es muy común encontrar en la literatura psicológica pronunciamientos como los siguientes: «aparte de la cuestión ontológica sobre la naturaleza y aun la existencia de una 'realidad' fija, singular y cognoscible, está también la cuestión epistemológica de si nosotros podríamos incluso conocer/reconocer tal realidad si siquiera existiese» (McGrath \& Johnson, 2003, p. 34); «...existen múltiples realidades construidas... más que una única realidad verdadera» (Ponterotto, 2005, p. 130); los hechos son «artefactos del proceso de observarlos» (Rapport, 2004, p. 101). Por supuesto, no falta la insistencia en la noción kuhniana de que los paradigmas su suceden unos a otros debido únicamente al peso de factores extracientíficos (e. g., Willig, 2013). Nosotros argumentamos que existe una diferencia fundamental entre, por un lado: (a) el carácter socialmente contextualizado de los llamados «eventos psicológicos», y por el otro, la pretensión de que todo conocimiento acerca de tales eventos (y de la realidad en general); (b) el producto de ciertas transacciones entre los observadores; (c) interpretación o percepción subjetiva, determinada total y únicamente por las circunstancias del observador; (d) el resultado de la acción de fuerzas sociales que presionan sobre la actividad científica; (e) el producto de una visión paradigmática asumida sobre bases no cognoscitivas o racionales, etc. Se puede estar de acuerdo con (a), que es compatible con una filosofía realista y objetivista, pero ello no necesariamente obliga a aceptar también los demás puntos, los cuales niegan abiertamente la posibilidad de conocimiento objetivo y con ello, la posibilidad de establecer al menos algunos hechos en una disciplina de tanta importancia no sólo teórica sino también práctica para el ser humano como es la psicología.

El constructivismo y el relativismo comienzan con la premisa, basada en una constatación común, de que cada individuo sostiene una visión de la realidad que es distinta de la de cualquier otro. Tal premisa no es por completo verdadera, ya que en muchos aspectos particulares puede no haber diferencias de opinión o percepción entre los individuos. Pero lo importante del constructivismo y el relativismo no es tal premisa, sino la conclusión que se pretende obtener a partir de ella: que existen múltiples realidades. Planteamos dos primeras objeciones a tal conclusión. Por un lado, lejos de servir a la causa constructivista-relativista, la constatación de tal diversidad de percepciones de la realidad apoya más bien una filosofía objetivista. Por otro lado, aun si la premisa mencionada fuera cierta para todo aspecto posible de la realidad, ella no implica que haya múltiples realidades; es decir, que no sea posible evaluar la validez relativa de tales visiones individuales.

Consideremos, por ejemplo, la observación frecuente de que los problemas elegidos en muchas áreas de investigación, e incluso los supuestos que subyacen a muchos conceptos centrales en dichas áreas, están principalmente vinculados a las necesidades y percepciones de los individuos del género masculino; cuando no están, incluso, diseñados para justificar la subordinación de las mujeres (Potter, 2006). La protesta de ciertos sectores autodenominados feministas ha sido muy útil para ganar conciencia acerca de esta inequidad (Stanley, 2013). Tal protesta ha prestado un servicio eminente a la causa de la objetividad (más bien que al relativismo) al resaltar la necesidad de reconocer ciertos sesgos en los problemas seleccionados como temas de investigación e incluso en la perspectiva conceptual desde la cual se abordan. Además, este reconocimiento no obliga a admitir que existen múltiples realidades. Que cada género tenga sus prioridades sólo significa que los problemas de investigación y sus marcos conceptuales deben diversificarse y extenderse; es decir, significa que la realidad es más compleja en sus componentes y estructura de lo que suponíamos. Y por supuesto, deberíamos ser capaces de llegar a una visión correcta de esa complejidad. Como veremos, el hecho de que el género o cualquier otra circunstancia del investigador puede sesgar sus observaciones y razonamientos, no excluye la posibilidad de alcanzar, por lo menos algunas veces, conclusiones objetivas. $\mathrm{O}$, por lo menos, no es impedimento para intentarlo. 
De hecho, es en interés de esos mismos sectores sociales excluidos o subordinados demostrar que su condición no es una construcción relativa a ciertos observadores, sino un hecho objetivo, real. La pertenencia a un género puede ser causa de ciertos sesgos, pero esos sesgos pueden ser descubiertos y por tanto, ni tal circunstancia ni ninguna otra posible fuente de sesgos es justificación suficiente para argumentar en favor del relativismo.

Por supuesto, al margen del argumento de que el científico es una víctima de los sesgos que inevitablemente afectan a todo ser humano, se ha dicho también muchas veces que no existe un punto privilegiado de observación desde el cual comparar las teorías con la realidad "tal como ésta es realmente». Si la objetividad consiste en lograr observaciones sin sesgo alguno y desde esa posición privilegiada (e. g., MacKinnon, 1991), entonces la objetividad no existe. Pero nótese que esto ya no es hablar de objetividad, sino de algo que, en efecto, no existe, a saber, omnisciencia. Pocos científicos, si alguno, creerán que ser objetivos es ser de alguna manera omniscientes. Tal concepción de la objetividad puede quizás adscribirse a ciertas formas ingenuas de empirismo, pero nosotros estamos aquí comenzando con una idea muy distinta de objetividad. Una que reconoce la posible existencia de sesgos en las observaciones y el pensamiento y que admite, en efecto, que no contamos con tal punto privilegiado de observación. Nuestro propósito es mostrar que aun en tales condiciones el constructivismo y el relativismo no son una consecuencia obligada. Es decir, que aun admitiendo dichas limitaciones, es posible establecer que existe un mundo objetivo y que podemos aprender al menos algunas verdades acerca de ese mundo, ya que los sesgos pueden ser controlados y dichas verdades siempre están limitadas a lo que nos revela la evidencia disponible. Primero se explicará con algo más de detalle el propósito del presente trabajo y a continuación se examinará hasta qué punto es obligatorio que las limitaciones señaladas y algunas otras conduzcan a una visión constructivista o relativista de la realidad.

\section{El problema}

Existen múltiples miradas para entender la objetividad en el campo científico (Agazzi, 2014; Daston \& Galison, 2007; Longino, 1990; Sceski, 2007). La preocupación fundamental en este trabajo es justificar la posibilidad de la objetividad del conocimiento científico, desde la visión aquí adoptada, es decir, la objetividad como posibilidad de evaluar el valor de verdad de un enunciado con base en evidencias y argumentos. Conocimiento objetivo es aquel conocimiento acerca de un mundo real y que está constituido por enunciados cuyo valor de verdad puede ser evaluado de esa manera. ¿Es posible obtener conocimiento de esta calidad? El propósito, por tanto, es restablecer en la disciplina psicológica la confianza, al parecer perdida o por perderse, en la objetividad.

Hagamos una lista de algunos argumentos que parecen descartar por completo la posibilidad de conocimiento objetivo:

1. Toda captación de la realidad es necesariamente una construcción del individuo. La realidad es enteramente construidasobre la base de las percepciones, pensamientos y acciones de un observador. Por tanto, o no es posible concluir que existan entidades independientes de, o preexistentes a, un observador o no es posible determinar en qué medida dichas construcciones corresponden a una realidad.

2. No existen hechos auténticos. Los «hechos» son el producto de transacciones entre los observadores, no el resultado de las evidencias.

3. Aun si existiera una realidad definida y pre-existente a un observador, éste es inevitablemente sesgado en sus percepciones y juicios por una serie de factores tanto personales como histórico-sociales.

4. Ciertos factores externos, como las circunstancias histórico-sociales, influyen decisivamente en la aceptación o rechazo de los hechos o las teorías. 
5. Las teorías e incluso los hechos son relativos a un marco conceptual (e. g., paradigmas) y, como en el caso anterior, no existen criterios objetivos para comparar tales marcos conceptuales. Por ejemplo, se dice que los paradigmas son inconmensurables.

6. Como sumario a partir de los puntos anteriores, las teorías son aceptadas o rechazadas de acuerdo con criterios no esencialmente cognoscitivos $\mathrm{u}$ «objetivos», sino enteramente irracionales o «subjetivos».

En suma, no hay manera de establecer verdades acerca del mundo y, por tanto, la aceptación o no de ciertos enunciados acerca de ese mundo depende de factores no racionales, como los factores psicológicos del observador o las condiciones socio-políticas en que está inmerso. Esto es escepticismo puro y lo que se puede llamar pesimismo epistémico.

En lo que sigue, se aborda críticamente cada uno de estos argumentos en el orden en que han sido presentados. Antes, formularemos las siguientes dos precisiones. En primer lugar, en cierto sentido o al menos en parte, los anteriores argumentos contienen algo de verdad. Por ejemplo, los hechos científicos no se presentan al ojo desnudo del investigador, sino que comúnmente son obtenidos mediante ciertas operaciones experimentales y representados con sustento en algún sistema conceptual. Todo observador y todo razonador se encuentran limitados por factores biológicos y sus observaciones y razonamientos pueden verse afectados por sus condiciones psicológicas y sociales. Asimismo, es absolutamente correcto que la percepción y el conocimiento son fundamentalmente conceptuales y por tanto, que lo que se conoce depende del marco conceptual del observador. Y por último, también es posible que en ciertos momentos, determinados factores irracionales pesen más que las evidencias y los argumentos - cuando los hay - en las decisiones científicas. Los constructivistas y relativistas pretenden obtener ventaja de lo que hay de cierto en todo esto. Hay que dejar en claro que un objetivista no negaría las partes verdaderas de los argumentos anteriores. El objetivista las considera como premisas que no necesariamente conducen a conclusiones constructivistas o relativistas.

En segundo lugar, es necesario agregar un breve comentario sobre la relación entre los conceptos de objetividad y verdad. Para Collier (2003) la palabra objetividad hace referencia simplemente a lo que es verdadero independientemente, incluso, de si alguien puede o no comprobarlo. Que la tierra es el tercer planeta en distancia al sol es un hecho objetivo en este sentido. Esta definición sustenta el postulado de objetividad ontológica. El término verdadero se usa aquí como equivalente a real, no como denotando solamente un atributo de los enunciados. Por otro lado, la objetividad epistémica se refiere, más bien, a la posibilidad de evaluar el valor de verdad de los enunciados. Al respecto, Collier (2003) señala que un juicio objetivo es aquél exclusivamente causado por su objeto (p. 135). Brown (2001) concuerda: Una persona es objetiva cuando se basa en «buenas» razones y evidencia para aceptar un enunciado (p. 102).

Sin embargo, Brown (2001) también advierte que objetividad no es lo mismo que certeza o verdad. La objetividad tampoco implica infalibilidad (Collier, 2003). Desde un punto de vista científico, es imposible hacer referencia a la verdad en un sentido absoluto. En realidad, todos sabemos que las proposiciones científicas sólo son provisionalmente verdaderas y lo son sólo en función de las mejores evidencias y razones disponibles en un momento histórico determinado. Así se dice que el enunciado «algunas enfermedades de los seres humanos son causadas por microorganismos» es verdadero, en tanto que «algunas enfermedades del ser humano son causadas por los demonios» es falso, sólo en el sentido de que se cuenta con buenas razones y evidencias para creer en el primero, pero no para creer en el segundo. Demandar a la ciencia la producción de certezas o verdades absolutas siempre 
ha sido algo extraño a una visión objetivista; sin embargo, algunos creen que criticar la búsqueda de «verdades absolutas» es algo que llega recién con la post-modernidad (e. g., Méndez, 2000).

\section{Constructivismo}

Se analiza la primera de las cuestiones arriba señaladas. El ser humano elabora construcciones mediante las cuales organiza su interacción con la realidad. Pero, ¿reflejan en verdad estas construcciones algo real? La cuestión central del constructivismo filosófico en general no es que el ser humano formule construcciones, sino que éstas no reflejan ninguna realidad independiente de, o preexistente a, un observador; por ejemplo, cuando se dice que dichas construcciones no dependen de alguna mejor comprensión de la realidad, sino de diversos factores sociales (Green \& Thorogood, 2004). En esta sección evaluaremos tal pretensión, la cual también puede expresarse mediante la afirmación de que los hechos son siempre hechos generados por la actividad de un observador.

Existen diversos argumentos de este tipo. Una postura radical afirmaría que, a lo sumo, sólo hay entidades indiferenciadas a las cuales nuestra percepción (con ayuda de un marco conceptual o de otros medios) impone un orden y significado. Así, no hay propiamente objetos definidos, salvo en nuestra percepción. Filósofos como Rorty y Goodman (como se citó en Boghossian, 2006) han argumentado en favor de esta postura radical. Por ejemplo, Gotman propuso el argumento de que los objetos son como las constelaciones que «vemos» cuando unimos un grupo de estrellas con líneas imaginarias. Así, los objetos existen solo como construcciones que son introducidas por el individuo, pero que no corresponderían a ninguna realidad externa a su percepción o pensamiento. Por esta misma razón, tales construcciones son arbitrarias en el sentido de que no existen criterios para determinar cuál es la constelación «correcta»; es decir, que todas las posibles maneras de trazar constelaciones son igualmente válidas. Sin embargo, como observa Boghossian (2006), aun cuando las constelaciones son construcciones, las estrellas así agrupadas no lo son. Es decir, no todo en el mundo son realmente entidades construidas en el sentido señalado. E incluso cuando son construidas, lo son sobre la base de entidades reales.

Una versión constructivista parecida a la anterior sostiene que las entidades no son los objetos concretos observados, sino las categorías que se elaboran para agruparlos. Por supuesto, dichas categorías son construidas. Por ejemplo, hay individuos que presentan cierto estado de salud, pero no existe algo así como una entidad llamada tuberculosis. Esta existe como una categoría construida por un observador. Del mismo modo, Koch observó algo a través del microscopio, pero no existe el así llamado bacilo de la tuberculosis independientemente de la categorización de los objetos vistos (y, por supuesto, en este sentido, tampoco existía el bacilo de la tuberculosis antes de que Koch formulara tal categoría a partir de sus observaciones). Por tanto, las enfermedades, los microbios y en general, los objetos y eventos que constituyen el mundo son construidos como categorías, las cuales, como es claro, existen sólo desde el momento en que se observan las entidades concretas que son de este modo categorizadas. Sin embargo, el problema no es que se construyan categorías, pues construir categorías es un medio para organizar una gran cantidad de información. Lo más importante es que aunque las categorías son construidas, los hechos y objetos a los que se refieren son reales. La situación es análoga a la del ejemplo de las constelaciones. Este punto quizá se aprecia mejor con el siguiente ejemplo: un campesino usa un solo nombre para referirse a entidades que un biólogo encuentra como miembros de dos o más especies. Aunque se podría decir que cada uno de estos individuos tiene una distinta visión de la realidad o que ellos «construyen» la realidad de diferentes maneras, lo que construyen son solamente sus respectivos sistemas de categorías, pues no hay duda de que 
dichos distintos sistemas se refieren a las mismas cosas, las cuales son reales e independientes de los individuos en cuestión.

Muchos constructivistas concordarán en que la acción de categorizar en sí misma no involucra constructivismo, y por tanto, pondrán énfasis más bien en el hecho de que tales categorías no representan nada real. Demostrar que esto es lo que sucede con los hechos científicos es lo que se encuentra en la agenda del llamado «programa fuerte» de la sociología de la ciencia, es decir, la sociología constructivista de la ciencia. Desde esta sociología, las entidades concretas que las categorías científicas pretenden agrupar no existen realmente y dichas categorías son propuestas solamente como resultado de ciertas transacciones entre los observadores o con fundamento en motivos totalmente ajenos a la búsqueda de la verdad. Por tanto, no hay hechos científicos, en el sentido de hechos «reales», sino solamente hechos «negociados» entre los investigadores. Así, pues, según Cole (1998) esta postura considera a la naturaleza como determinada por el consenso social, en vez de ser éste último determinado por aquélla.

No obstante, hay aquí un agudo problema lógico del cual los sociólogos constructivistas de la ciencia no pueden escapar. Como Brown (2001) y muchos otros han hecho notar, para los constructivistas los científicos no están descubriendo nada; sin embargo, los propios constructivistas sí parecen estar actuando objetivamente al «descubrir» que los científicos no descubren nada. Si los constructivistas deciden realizar su investigación apegados a todos los estándares de la buena práctica científica, y por tanto, asegurando la objetividad de sus conclusiones, ellos podrían reclamar entonces que sus resultados son legítimos y válidos, pero a la vez tendrían también que admitir la validez de dichos estándares, entre ellos, el examen objetivo de las evidencias, como un medio para arribar a conclusiones confiables. Así pues, se puede aplicar a toda la sociología constructivista de la ciencia el juicio que formuló Isambert (1985) acerca de uno de sus representantes más conspicuos, cuando señaló que Latour apelaba a una razón que él mismo había repudiado.

Pero esta dificultad lógica no es el único problema en el trabajo de los sociólogos constructivistas de la ciencia. Existe clara evidencia de que las conclusiones que defienden no están realmente apoyadas por sus observaciones. Cole (1992) ha documentado cómo ninguno de los estudios constructivistas de la ciencia demuestra que las «negociaciones» entre los investigadores podrían haber influido en el contenido específico de los resultados científicos. Por otro lado, como advierten Sokal y Bricmont (1997), un sociólogo puede percibir como un simple juego de poder lo que en realidad es una compleja red de razonamientos y procedimientos plenamente justificados, aunque sólo visibles para quienes tengan suficientes conocimientos del tema. Así, el «hecho científico» sólo puede ser percibido por personas especialmente entrenadas. Por ejemplo, como es mostrado por Brown (1994), un determinado procedimiento de bioensayo es aceptado por los investigadores, no como resultado de «negociaciones» en el laboratorio, sino a causa de restricciones reales, que lo hacen técnicamente el más apropiado. Este caso constituye un ejemplo del principio más general de que los «hechos» son reconocidos como parte de ciertas prácticas con las cuales uno debe estar familiarizado para percibir tales hechos; pero dichas prácticas consisten en acciones, más o menos complejas o especializadas, realizadas respecto de la realidad; no son simplemente las confabulaciones de investigadores ansiosos de poder que inventan la realidad para satisfacer su ambición. Por supuesto, la crítica a la sociología constructivista de la ciencia se refiere a su implicación de que el hecho científico es en su naturaleza nada más que un producto resultante de fuerzas sociales, pero esto no impide reconocer que algunos supuestos «hechos científicos» sí pueden tener ese carácter.

También se ha dicho que las propiedades de los objetos o eventos no son intrínsecas a éstos, sino que 
dependen del instrumento o métodos con que se observan. Esta es una postura que algunos consideran consistente con la física cuántica: Según Bohr (1958) y Heisenberg (1958), las partículas subatómicas no tienen propiedades definidas mientras no interactúen con un instrumento de medición. Sin embargo, no todos los físicos comparten esta creencia y de este modo, hay poca base científica real para esta forma de constructivismo (e. g., Baggott, 1993; Norris, 2003).

En suma, un hecho físico o biológico es siempre una categoría de entidades o de eventos que existen independientemente de un observador. Se asume que dichas categorías engloban entidades reales en la medida que no se introducen de manera arbitraria, sino con sustento en evidencias y argumentos. Categorías así generadas son las que comprenden el conocimiento objetivo.

Por otro lado, reconocer el carácter convencional de los eventos psicológicos y sociales no significa avalar las posiciones constructivistas examinadas en esta sección. Los eventos psicológicos y sociales son prácticas convencionales y tales convenciones varían según tiempos y lugares, pero el individuo que participa en dichas prácticas no las ha generado mediante algún acto de observación ni ellas son producidas por la interacción de alguna entidad con determinados medios de observación (como se pretende que ocurre con las propiedades de los objetos cuánticos) ni tampoco tales prácticas existen sólo en los artículos producidos (mediante «negociaciones») por investigadores ambiciosos interesados en acumular publicaciones. Tales hechos son objetivos en el sentido de que dichas prácticas, una vez que se establecen, adquieren autonomía y un estatus de realidad en muchos aspectos comparable al de los eventos naturales. Por ejemplo, y teniendo a mano todas las evidencias del caso, nadie puede cuestionar la realidad de un acto matrimonial realmente efectuado en determinado momento y lugar, ni tampoco puede seriamente cuestionar que sea posible, hasta cierto punto, determinar las causas y consecuencias de dicho acto. Del mismo modo, aunque hace falta algún contacto previo con ciertas experiencias, directas o mediante instrucción, para que podamos percibir determinadas circunstancias como «guerra» o como «pobreza», quienes sufren esas circunstancias ciertamente no sufren por causa de nuestras construcciones. O cuando una mujer es golpeada por su pareja, es ella quien experimenta el dolor, ya sea que tal hecho se categorice o no como «violencia doméstica», etc.

\section{Relativismo basado en factores empíricos}

Las condiciones personales, como el género o los antecedentes culturales, así como las necesidades e intereses particulares, generan determinadas actitudes, creencias y valores en el investigador. Estas condiciones — se ha dicho muchas veces- no sólo afectan la elección de los temas a investigar, sino también la perspectiva conceptual que se adopta y, por consiguiente, incluso, la interpretación de los datos. El trabajo del científico es también afectado en el mismo sentido por factores «externos», es decir, histórico-sociales y políticos. Todo esto, sin duda, constituye un hecho empírico, objetivo e innegable. La pregunta no es si tales condiciones realmente influyen en la ciencia, sino si esta influencia impide en todo momento establecer al menos algunas verdades acerca del mundo. Lo que afirma el relativismo que llamaremos empírico no es solamente que las condiciones señaladas generan determinados sesgos en las observaciones o razonamientos del investigador, sino que tales sesgos impiden tener en consideración criterios objetivos para valorar dichas observaciones y razonamientos (Vanderstoep \& Johnston, 2009).

En primer lugar, no hay realmente nada postmoderno en lo que afirman los relativistas, pues al menos ya desde los griegos se sabía que las observaciones pueden verse influidas por muchos factores personales y sociales (Kirk, 1999). Sin embargo, aun cuando quien conoce esté sujeto a muchas fuentes personales y sociales de sesgo, ello 
no elimina la posibilidad de arribar a algunas conclusiones bien fundadas o válidas acerca del mundo, y que, por tanto, trascienden las circunstancias personales del investigador. Es por esta razón que incluso los subjetivistas arman muchos de sus argumentos confiando, por ejemplo, en las partes bien establecidas de la ciencia, como las predicciones de ciertas teorías o la evidencia experimental. El hecho de admitir la posibilidad de tales sesgos ya es un reconocimiento de que es posible establecer algunas verdades sobre la base de evidencias que son incontrovertibles. Un objetivista no afirma que siempre sea posible llegar a determinar la verdad o el valor de una evidencia; lo que sostiene es que sí es posible hacer todo esto al menos algunas veces (Boghossian, 2006; Brown, 2001). El relativista, en cambio, niega por completo tal posibilidad.

Los sesgos en las observaciones y juicios constituyen fenómenos bien conocidos por el psicólogo experimental. Se sabe que diversas variables tanto del observador como del estímulo pueden afectar la percepción. Hasta un relativista deberá aceptar estos hechos como objetivos, pues, en realidad, la parte más importante de sus argumentos descansa en ellos. El problema consiste en si es posible determinar cuál es la percepción «correcta». La regla general es que la varianza en la percepción cambia directamente con la fuerza de los factores psicológicos e inversamente con el monto de información que porta el estímulo. A determinado nivel en el balance entre los factores psicológicos y del estímulo, la varianza en la percepción dependerá de otros factores, como el marco conceptual del observador, a lo que nos vamos a referir más abajo. Ahora bien, excepto bajo condiciones psicológicas o estimulares extremas, la percepción por lo general es la correcta, en el sentido, por ejemplo, de que al observar un objeto, es posible distinguir si se le percibe distorsionado o como realmente es.

En otras palabras, las convicciones teóricas (o la posición social, etc.) del científico no pueden sesgar sus observaciones hasta el punto de impedirle constatar lo evidente (aunque sí pueden, a veces, conducirle a querer ocultar o distorsionar los hechos o a negarse a aceptar determinadas implicaciones de estos hechos). Como observa Brown (2001) las razones y las evidencias, por lo menos algunas veces, pueden justificar plenamente nuestras creencias. Además, y esto es lo más importante, podemos percatarnos del hecho, totalmente objetivo, de la posible influencia de los factores personales o sociales sobre nuestras observaciones y juicios (tal como ha sido puesto de relieve por la psicología experimental). Es por ello que es también posible reducir (si no eliminar por completo) dicha influencia. La objetividad, en la visión del científico, es el esfuerzo por identificar los posibles sesgos y controlarlos (Collier, 2003).

Por otro lado, los factores externos tienen también un impacto innegable en la investigación científica y en las decisiones del investigador. Por ejemplo, se ha hecho notar muchas veces que dependiendo de quién detenta el poder se toman decisiones acerca de cuáles son las prioridades en investigación y, por lo tanto, qué contenidos de conocimiento se producen. Esos factores también influyen en la aceptación o rechazo de los hechos y las teorías (Harding, 1999). Tales factores pueden influir en la conducta del científico individual, pero también en la actividad científica organizada como actividad colectiva. Como ilustración de ese impacto podemos recoger los comentarios de Haely (2008) sobre el caso de Cyril Burt. El problema no consiste sólo en la falsificación posteriormente demostrada, sino también en que durante décadas nadie se preocupó por ahondar críticamente en los resultados de Burt. Según Haely (2008), el clima político e ideológico de esa época fue favorable a la aceptación acrítica de aquellos resultados. Así, pues, es de suponer que determinados intereses se introducen más o menos subrepticiamente — a veces abiertamente — en el juicio de los expertos en determinada materia, no permitiéndoles estimar de modo más crítico el valor de determinadas evidencias. 
Ahora bien, el impacto de los factores externos es real e innegable, es un hecho objetivo, no construido ni relativo a algún observador. Y ese impacto puede llegar a tener un peso considerable respecto de las ideas que son aceptadas o rechazadas. Que se trata de algo real es ilustrado, por ejemplo, en el hecho de que incluso quienes simpatizan con la idea del carácter negociado de los hechos científicos, acaban usando su poder para imponer tal idea en el ambiente académico (Cole, 1998). El clima político puede auspiciar u obstaculizar el tratamiento de ciertos problemas, y con ello, determinar de qué evidencias se dispone en un momento dado y, por tanto, a qué conclusiones se puede llegar; pero también puede ejercer cierto grado de presión en cuanto a qué se publica o se enseña. Estos hechos, objetivos, no constituyen sino uno de los muchos factores externos que contextualizan el desarrollo científico.

Pero una vez que se ha adoptado la decisión de investigar determinados problemas, la validez de los resultados puede determinarse de modo relativamente independiente de los motivos políticos. En el caso de Burt, por ejemplo, el nuevo clima político condujo a un cuestionamiento de sus conclusiones y, con ello, a la revisión de sus resultados; pero para nosotros lo importante de ese episodio es que a partir de esa revisión fue posible establecer algunos hechos en relación con tales resultados. Así, el ambiente político actúa como una fuerza que a veces presiona en relación con qué asuntos investigar, qué resultados enseñar y publicar, pero no puede afectar la validez de los hechos.

Es también por esta razón que no se puede apoyar ni desacreditar teorías o resultados experimentales sólo con base en los motivos e intereses que supuestamente estén detrás de ellos. Por ejemplo, Brown (2001) cita trabajos según los cuales Pasteur fue movido por sus propias convicciones políticas a realizar los experimentos que demostraron la imposibilidad de la generación espontánea. Pero a ningún científico se le ocurriría cuestionar la objetividad o la validez de los resultados presentados por Pasteur a partir simplemente de tales argumentos históricos. Del mismo modo, Hessen (2009) explica detalladamente cuál era el contexto social y económico que determinó el tipo de problemas que Newton abordó en su obra, pero sería poco sabio concluir que la validez de la mecánica newtoniana depende de, o se limita a, dicho contexto.

Cuando el interés en la verdad no es solamente epistémico (esto es, el interés en conocerla), sino también moral (esto es, el interés en defenderla), aun cuando ello signifique enfrentarse a determinados intereses, los mejores recursos siguen siendo las evidencias y los argumentos. Por ello, aunque ciertas élites intenten imponer teorías erradas, siempre existe la posibilidad de crítica y de debate sobre la base de razones y evidencias. En suma, puede haber muchos intereses involucrados en la aceptación o rechazo de los hechos o las teorías científicas, pero los hechos y las teorías aún pueden ser validados en forma independiente de aquellos intereses. Y el único recurso de valor en el debate entre teorías rivales tenga o no tal debate motivaciones políticassiempre serán los argumentos y las evidencias.

\section{Relativismo conceptual}

Llegar a ciertas verdades depende de la disponibilidad de buenas evidencias, pero, ¿qué evidencias son aceptables? Se ha repetido innumerables veces que incluso la observación depende de un marco conceptual. Y por supuesto, no sólo las observaciones, sino todo planteamiento que aspire a ser conocimiento. El conocimiento, ya sea en la forma de simple percepción o de teoría, está limitado a las capacidades biológicas del individuo cognoscente y a su sistema conceptual. Las limitaciones biológicas son obvias e indiscutibles: En ausencia de instrumentos sólo podemos ver lo que nuestros ojos pueden ver. Pero el impacto de las limitaciones conceptuales es igualmente importante. 
Pero, ¿qué entendemos por marco conceptual? Un marco conceptual es una red de conceptos. Estas redes existen en distintos niveles. Por ejemplo, un marco conceptual puede consistir en las propiedades asignadas a determinada clase de eventos. Otro marco, más básico, puede consistir en las definiciones establecidas para dichas propiedades. La ubicuidad de los marcos conceptuales implica que, en ciencia, como en cualquier otra actividad, siempre hay interpretación. En ciencia, la interpretación se refiere a distintas circunstancias, por ejemplo, como categorización del contenido de las impresiones sensoriales (por ejemplo, la respuesta a las lecturas que los instrumentos arrojan) o como conjeturas particulares acerca del evento observado. En ambos casos siempre está implicado un marco conceptual como guía para tales interpretaciones. Pero todo esto es muy distinto de los sesgos de interpretación generados por la influencia de factores personales, sociales o políticos. A menos que se considere, como hacen muchos relativistas, que los propios marcos conceptuales son elegidos en función de tales factores personales, sociales y políticos. Lo que nos devuelve a la cuestión de cuánto pueden las evidencias determinar las conclusiones por encima de tales sesgos.

La posición objetivista admite que toda observación y razonamiento se expresa en términos de algún marco conceptual. En realidad, ningún objetivista igualará objetividad con empirismo ingenuo. No hay necesidad de recordarle a un objetivista que las percepciones del investigador dependen de su marco teórico (Corbetta, 2003). Todos estamos de acuerdo, y los objetivistas en primer lugar, en que todo conocimiento existe como una red de conceptos. Aun la observación es conceptual (Feist, 2006).

Por tanto, la pregunta fundamental para optar entre objetividad y relativismo no es si las observaciones dependen o no de un marco conceptual. Es decir, si hay o no interpretación. La pregunta fundamental es más bien la de cuán justificado está un determinado marco conceptual y, por tanto, determinada interpretación. El relativismo niega que sea posible hallar tales justificaciones, por tanto, un marco conceptual es tan bueno como cualquier otro. Y como todo marco conceptual constituye el medio para representar la realidad, desde el relativismo se concluye que existen múltiples realidades o múltiples representaciones válidas de la realidad, incluso aunque sean contradictorias entre sí. Veamos si la naturaleza conceptual del conocimiento constituye un argumento a favor del relativismo.

Como se dijo, un nivel conceptual es el de las definiciones. Ahora bien, las definiciones constituyen el sistema conceptual básico con el cual se formula cualquier pretensión de conocimiento. Según nuestra definición, la objetividad no requiere independencia de todo posible marco conceptual. Como ya dijimos, el conocimiento debe formularse en términos de algún marco conceptual. El problema de la objetividad de un discurso no consiste en justificar las definiciones de los conceptos con que se formula, sino en si es posible evaluar el valor de verdad de los enunciados que conforman dicho discurso. Un nivel conceptual relacionado con el de las definiciones es el de los sistemas de clasificación. Como se dijo, los seres humanos construyen sistemas de categorías para organizar la información y, basados en ello, se puede decir que se «observan» cosas distintas, aunque se esté mirando el mismo objeto, dependiendo del sistema de categorías del observador. Hanson (1971) concuerda: «Observar es categorizar». Este autor propuso un ejemplo ilustrativo: a la vista de un mismo objeto, un observador lo ve como un simple tubo de metal y el otro como un telescopio. El ejemplo tiene la intención de mostrar hasta qué punto la percepción puede verse influida por el marco conceptual del observador. Pero el ejemplo no avala el relativismo, ya que el objeto puede ser a la vez, y sin contradicción, un tubo de metal y un telescopio. Es decir, no existe contradicción entre ambos marcos conceptuales.

Otro nivel conceptual comprende los enunciados construidos sobre la base de un sistema de 
definiciones y clasificaciones. A tales marcos conceptuales se les llama a veces teorías. Por supuesto, es común en diferentes campos científicos que existan teorías que se contradicen entre sí, aun ocupándose del mismo dominio empírico. El relativista afirma que distintas teorías, incluso aunque sean incompatibles entre sí, son igualmente válidas. El objetivista, en cambio, se vale simplemente del principio de no contradicción: dos teorías que se contradicen una a la otra no pueden ser ambas verdaderas. Nótese que hablar de teorías compatibles o incompatibles entre sí, ya implica el reconocimiento de que existe un lenguaje, un marco conceptual, más fundamental, que permite hacer estas comparaciones. El objetivista cree que por mucha contradicción que exista entre dos teorías, siempre es posible discernir entre ellas con fundamento en evidencia y argumentos formulados en ese nivel conceptual más básico.

Antes de enfocarnos en esa posibilidad, mencionaremos brevemente la posición de ciertos relativistas que otorgan el mismo nivel de validez a diferentes manifestaciones de otro tipo de marco conceptual, a saber, lo que podríamos llamar cosmovisiones. Boghossian (2006) comienza su crítica del constructivismo y del relativismo citando el siguiente caso. Se puede decir que los pueblos aborígenes de América vinieron de Asia, como se puede también afirmar que los pueblos aborígenes de América fueron engendrados de la tierra. Estas expresiones se contradicen entre sí, ambas no pueden ser a la vez verdaderas. Pero si se asume la validez simultánea de las cosmovisiones científica y mitológica, entonces se puede aceptar que ambas afirmaciones son verdaderas, por supuesto, cada una en el marco de su respectiva cosmovisión. Todo ello, además, se ve fortalecido cuando se asume que tampoco hay criterios objetivos para evaluar las cosmovisiones. Ahora bien, reconociendo el derecho a la existencia de todas las expresiones culturales, la diferencia entre el objetivista y el relativista es la siguiente. El primero distingue entre diferentes modos de conocimiento y sus respectivas funcionalidades sociales. Así, el conocimiento científico y el mitológico no son comparables y por tanto, sólo el primero puede proveernos de respuestas acerca de los hechos del mundo real. El relativista, en cambio, coloca a ambos en pie de igualdad en relación con las preguntas sobre el mundo real, ya que para él no existe un criterio único de «validez» del conocimiento. La esencia de esta forma de relativismo es la validación de distintas y contradictorias cosmovisiones y racionalidades para juzgar las afirmaciones acerca del mundo.

Dejemos de lado tal forma extrema de relativismo, para la cual no hay respuesta racional posible, y concentrémonos en lo que sucede en aquellos espacios en los que al menos hay acuerdo en la adopción de la cosmovisión científica, donde también hay ciertas inquietudes relativistas. Una idea relativista ampliamente comentada es la de los paradigmas científicos. Se afirma que los paradigmas son inconmensurables; es decir, son como distintos idiomas o lenguajes. Una traducción precisa de lo que se quiere decir en el marco de uno de ellos a los términos del otro, es imposible. Dos paradigmas son dos visiones distintas del mundo, el observador ve a través de su paradigma y los creyentes de paradigmas distintos verán distintas cosas aunque observen el mismo objeto. La inconmensurabilidad de paradigmas implica que no existen criterios objetivos para evaluarlos y llegar a una decisión sobre su aceptabilidad.

Sin embargo, algo que los relativistas usualmente pasan por alto en sus lecturas de Kuhn (1977) es que dicho autor no consideró los paradigmas como totalmente inconmensurables al punto de imposibilitar la comunicación entre los partidarios de paradigmas rivales (Andersen, Barker, \& Chen, 2006). Y es aquí donde cobra interés la idea de marcos conceptuales de distinto nivel. De este modo, los paradigmas son una clase de sistema conceptual, pero existen sistemas conceptuales más básicos comunes a distintos sistemas conceptuales de un nivel más alto. En algún punto de la regresión al mundo empírico, las teorías rivales harán contacto con un lenguaje 
común. Si la evidencia necesaria se formulara solamente en el marco de las teorías puestas a prueba no sería posible utilizarla para decidir entre dichas teorías. Pero siempre es posible formular la evidencia en un lenguaje más empírico, aceptable por todos los involucrados en la discusión. Los paradigmas y las teorías en general pueden ser evaluados con base en evidencias que puedan formularse en un lenguaje común. De este modo, existe, por ejemplo, clara evidencia que favorece a Einstein sobre Newton en relación con la acción de un campo gravitatorio intenso sobre la trayectoria de la luz. O, citando otro ejemplo, si nunca tenemos evidencias para decidir entre una u otra teoría, ¿cómo podríamos afirmar que Kepler y Galileo demostraron que Aristóteles estaba equivocado?

\section{Sobre la racionalidad y objetividad de la decisión científica}

Hemos señalado que, para algunos relativistas, la aceptación y el rechazo de las teorías nunca se basan en evidencia objetiva; bien sea porque los sesgos observacionales no permiten obtener tal clase de evidencia, o bien porque, habiéndola, el peso de las necesidades e intereses de los investigadores se impone por encima de ella. De este modo, los intereses y las necesidades, y no la evidencia, serían siempre los jueces en última instancia en la decisión de aceptar o rechazar teorías. Pero hemos señalado también que por lo menos algunas veces la evidencia constituye el motivo fundamental para dicha decisión. Decimos que tales decisiones son racionales, cuando se asumen sobre la base de evidencia relevante. Pero al mismo tiempo decimos que tales decisiones son objetivas en tanto ellas no son el simple resultado de los factores psicológicos o sociológicos que tanto enfatizan los teóricos constructivistas y relativistas.

Pero la evidencia no es el único fundamento para una decisión racional. En muchas ocasiones los científicos han aceptado o rechazado teorías aun sin contar con evidencia relevante. ¿̇ndica tal conducta ausencia de racionalidad? Sólo se puede hablar de racionalidad cuando existen razones para una decisión. Si en determinados momentos los científicos tomaron tales decisiones en ausencia de evidencia, esto se debe a otras razones, igualmente válidas, como fundamento para tal conducta. Así, pues, en la decisión científica existe racionalidad y objetividad incluso cuando no hay evidencia suficiente, siempre que haya razones que respalden tal decisión.

Entre esas razones adicionales se encuentran los factores que Potter (2006) denomina «valores». Algunos de los valores discutidos por Potter (2006) tienen que ver con el impacto social que se espera obtener de los resultados. Por ejemplo, al decidir si acepta o no determinada hipótesis, el científico puede tener en consideración la importancia de no cometer un error, como por ejemplo, al asegurar que un fármaco es seguro y efectivo. Potter (2006) no niega que se pueda llegar a identificar determinadas verdades, así que la objetividad, como la entendemos, no está en cuestión; pero afirma que la meta de la ciencia no es solo identificar verdades, sino principalmente verdades que sean socialmente significativas.

Por otro lado, Potter (2006) menciona también ciertos «valores epistémicos» que son determinantes en la aceptación o rechazo de las teorías. Dichos valores consisten en determinados criterios, además de los empíricos, para juzgar las teorías. Es interesante notar que quienes se inspiran en Kuhn (1977) para destacar el papel de ciertos factores irracionales en la aceptación o rechazo de las teorías, ignoren que este autor reconoció finalmente que los científicos actúan sobre la base de ciertos criterios racionales para decidir entre teorías y describió cinco de ellos: capacidad para conducir a predicciones exactas, consistencia interna y externa, amplia cobertura de hechos, simplicidad y aptitud para conducir la investigación hacia nuevos hallazgos. Kuhn (1977) también explicó algunas de las dificultades que los científicos podrían encontrar al tratar de aplicar estos criterios en ciertos casos 
concretos. Pero son criterios que trabajan: Kuhn (1977) explica, por ejemplo, que el triunfo de la teoría de Copérnico se debió decisivamente a su mayor simplicidad frente a la teoría de Ptolomeo. Otros pensadores también han propuesto criterios racionales u objetivos para la decisión científica. Por ejemplo, Lakatos (1978) señala que esta elección no es una decisión afectiva entre paradigmas inconmensurables, sino una entre distintos programas de investigación según su grado de progresividad, es decir, su capacidad para generar predicciones interesantes. Por otro lado, según la propuesta de Laudan (1977) el científico elige aquella teoría que resuelve más problemas.

Es posible que en determinado momento no se cuente con la evidencia que corrobore las predicciones específicas de una teoría. Sin embargo, cuando ésta es más simple, resuelve más problemas o genera más predicciones que otras, será considerada mejor o más correcta que esas otras. Por ejemplo, un criterio poderoso es la capacidad de una teoría para incorporar fenómenos conocidos y resolver problemas. En este sentido, la decisión de aceptar la relatividad general dejando de lado la mecánica de Newton (y considerar la primera más verdadera que la segunda), aun antes de que se reunieran las evidencias pertinentes, era correcta en la medida que la primera explicaba fenómenos conocidos para los cuales la segunda no tenía ninguna explicación. Otros dos criterios que nos parecen igualmente racionales (y por ende, objetivos) son la consistencia interna y externa de la teoría y su capacidad para generar predicciones exactas. Teorías inconsistentes no pueden contener nada de verdad, salvo quizá solo en algunas de sus partes, y teorías que únicamente permiten predicciones inexactas son ellas mismas inexactas. En suma, la posibilidad de obtener evidencias y de contar con criterios objetivos para evaluar las teorías nos permiten concluir que la decisión científica no depende necesariamente de los factores sociales, psicológicos o políticos que los relativistas destacan tanto.

\section{Los paradigmas y el progreso científico}

Tradicionalmente se ha mantenido la idea de que la ciencia progresa, en el sentido de que el conocimiento científico es cada vez más profundo y verdadero. En suma, que hoy sabemos más de lo que sabíamos ayer acerca de nuestro mundo. Pero, ¿cuál es la esencia de este progreso? Este aumento de conocimiento no depende solamente del descubrimiento de nuevos fenómenos sino que también radica, quizá principalmente, en los cambios teóricos, como lo señalaron Einstein e Infeld (1986). Apenas se necesita hoy poner énfasis en ello. Sin embargo, con frecuencia no se entienden bien los alcances de tales cambios teóricos. Muchas veces se afirma, o se quiere dar a entender, justamente lo contrario, que dichos cambios consisten en la sustitución de unos conocimientos por otros, es decir, que no hay un verdadero avance o ascenso en el conocimiento. Si por «progreso» se entiende una mejora de nuestros conocimientos, entonces, se afirma, no hay verdadero progreso, precisamente porque no hay acumulación, sino sustitución de conocimientos. Y aún más, se insiste, sólo hay sustitución de un paradigma por otro, este último tan válido como el anterior. Agreguemos que esta idea corre al lado de aquélla otra de que los cambios paradigmáticos no obedecen a razones sino a factores sociales y psicológicos.

Sin embargo, no existe una contradicción entre la realidad de los cambios conceptuales y el progreso científico entendido como una mejora de nuestro conocimiento. Pero la única manera de comprender que ambos, cambio conceptual y a la vez progreso acumulativo, se implican mutuamente en vez de contradecirse es comprendiendo, en primer lugar, que dichos cambios conceptuales no tienen siempre porqué implicar rupturas dramáticas con el pasado y, en segundo lugar, que cuando se dan esas rupturas, siempre hay buenas razones para ello.

Por un lado, desde cierto punto de vista, cabe destacar la continuidad en el sentido de progreso 
acumulativo en el conocimiento. Según Elitzur (2005), la historia de la ciencia permite afirmar que las nuevas teorías siempre tienen un lugar incluso para los datos revelados en el marco de teorías anteriores. Esa misma línea de pensamiento es expresada por Einstein e Infeld (1986), cuando insisten en que las viejas teorías quedan incorporadas como casos particulares de las nuevas. Es importante mencionar que ellos hacen referencia a la idea de «dominio de validez» de una teoría. Por ejemplo, a la luz de la relatividad, la mecánica newtoniana no es enteramente verdadera; sin embargo, ésta puede considerarse una buena aproximación a la realidad tratándose de la mayoría de las acciones que llevamos a cabo en la vida cotidiana. De este modo, aunque la relatividad es un marco conceptual enteramente distinto de la mecánica newtoniana, no se puede negar los hechos que caen en la «región de validez» de esta última y de ello pueden dar fe los ingenieros que la usan a diario para diseñar desde edificios hasta vuelos espaciales.

Por otro lado, existe otra forma de progreso y es cuando descartamos los errores. El objetivista sostiene que el error puede ser detectado y que a partir de ahí conocemos mejor. Precisamente por esta razón es que podemos llegar también a la conclusión de que los distintos paradigmas no son igualmente aceptables y que, por tanto, la cuestión de decidir entre ellos no es simplemente un asunto de preferencias o de política. Los cambios conceptuales implican no sólo sustitución de unas ideas por otras, sino sustitución de unas ideas por otras mejores, es decir, verdadero progreso. En verdad, muchos filósofos parecen encontrar gran complacencia en cuestionar el carácter progresivo, no simplemente sustitutivo, del conocimiento científico, pero esa no es la actitud de quienes contribuyen a ese progreso.

\section{Conclusión}

A la luz de lo discutido, las respuestas del objetivista al reto del constructivismo y del relativismo son las siguientes:

1. El individuo elabora una imagen, o si se quiere, construcciones, de la realidad. Pero el objetivista asume que dichas construcciones son distintas de los objetos y eventos a los que ellas se refieren. Existe una realidad independiente de las construcciones elaboradas para representarla.

2. En algunos casos puede haber transacciones entre los observadores respecto de lo que debe aceptarse como «hecho». Pero no todo hecho científico es una consecuencia de tales transacciones. Existen razones teóricas y empíricas por las que un hecho científico es aceptado o no como tal. Y las transacciones mismas, cuando las hay, no son «construcciones», sino hechos auténticos.

3. Los sesgos son hechos reales, objetivos, no «construcciones» de un observador. Y como hechos reales, tienen un impacto real. De hecho, la necesidad y la posibilidad de controlar dichos sesgos constituyen un doble problema, pues tienen que ver tanto con las características como con la práctica del método científico.

4. Aunque la injerencia de los factores externos puede influir en la aceptación o rechazo de las ideas científicas, aún es posible, al menos en algunos casos, estimar la validez de dichas ideas, independientemente de dicha injerencia.

5. Los paradigmas no son por completo inconmensurables.

6. Como consecuencia de las consideraciones anteriores, se puede concluir que, al menos algunas veces, las teorías son aceptadas o rechazadas con base en criterios racionales, tales como las evidencias y determinados valores. 
Podemos resumir nuestros argumentos del siguiente modo. El conocimiento se formula siempre en el contexto de algún marco conceptual. Dichos marcos conceptuales (teorías, paradigmas, etc.) pueden discutirse, sobre la base de evidencias y argumentos, en términos de marcos conceptuales de nivel más bajo. Por otro lado, los sesgos observacionales o de juicio pueden ser detectados $\mathrm{y}$ controlados al menos algunas veces $y$, de este modo, es posible no sólo contar con evidencias, sino también establecer la validez de tales evidencias. Por tanto, dichas evidencias nos permiten, por lo menos algunas veces, establecer ciertos hechos acerca de la realidad. Los factores sociales, económicos y políticos que contextualizan la vida humana, incluyendo la investigación científica, pueden auspiciar u obstaculizar las decisiones acerca de qué investigar y publicar, pero no determinan por sí mismos la validez de los resultados.

Un breve comentario previo al cierre. Es paradójico constatar que haya profundas reivindicaciones sociales detrás de ciertas creencias relativistas y que tales creencias, sin embargo, no sean útiles para promover ninguna acción política. Muchos relativistas son conscientes de esta debilidad y se preguntan cómo actuar para generar cambios sociales si no puede afirmarse nada verdadero respecto de la falta de justicia o de libertad (Hicks \& Taylor, 2008). E incluso, es sorprendente que a la vista de tales dificultades, opten por relativizar el concepto mismo de cambio social y además concluir que el relativismo no es tan malo, frente al daño causado en el mundo por las «certezas absolutistas» (p. 72). Esa respuesta es lamentable por dos razones: primero, porque esquiva el cuestionamiento fundamental al relativismo y segundo porque, otra vez, está dirigida al blanco incorrecto, ya que lo que ella ataca no es la objetividad sino una pretendida omnisciencia en la que ningún científico cree.

Cada individuo tiene una percepción única de la realidad y esto es un hecho, es decir, es un aspecto objetivo de la realidad. La objetividad también implica tener en cuenta todas esas distintas percepciones en la búsqueda de lo que, sin embargo, deben ser las verdades que la ciencia persigue. Por tanto, como investigadores y educadores, nuestra visión debería ser siempre la del optimismo epistémico. Un optimismo que cree en el constante crecimiento del conocimiento humano como meta y como realidad. Más aun en un campo como la psicología, cuyas contribuciones son tan necesarias para el bienestar del ser humano.

\section{Referencias}

Agazzi, E. (2014). Scientific objectivity and its contexts. New York: Springer.

Andersen, H., Barker, P., \& Chen, X. (2006). The cognitive structure of scientific revolutions. Cambridge, RU: Cambridge University Press.

Baghramian, M. (2004). Relativism. New York: Routledge.

Baggott, J. (1993). The meaning of quantum theory. A guide for students of chemistry and physics. Oxford, RU: Oxford University Press.

Boghossian, P. A. (2006). Fear of knowledge. Against relativism and constructivism. Oxford, RU: Oxford University Press.

Bohr, N. (1958). Atomic physics and human knowledge. New York: Wiley.

Brown, J. R. (1994). Smoke and mirrors. How science reflects reality. New York: Routledge.

Brown, J. R. (2001). Who rules in science. An opinionated guide to the wars. Cambridge, MA: Harvard University Press.

Cole, S. (1992). Making science. Between nature and society. Cambridge, MA: Harvard University Press.

Cole, S. (1998). Voodoo sociology. Recent developments in the sociology of science. En P. R. Gross, N. Levitt \& M. W. Lewis (Eds.), The flight from science and reason (pp. 274-287) (4a. impresión). New York: The New York Academy of Sciences.

Collier, A. (2003). In defence of objectivity and other essays. On realism, existentialism and politics. London: Routledge. 
Corbetta, P. (2003). Social research. Theory, methods and techniques. London: Sage.

Daston, L., \& Galison, P. (2007). Objectivity. New York: Zone Books.

Einstein, A., \& Infeld, L. (1986). La evolución de la física (publicación original de 1936). Barcelona: Salvat.

Elitzur, A. C. (2005). What is the measurement problem anyway? Introductory reflections of quantum puzzles. En A. C. Elitzur, S. Dolev \& N. Kolenda (Eds.), Quo vadis quantum mechanics? (pp. 1-5). New York: Springer. doi: 10.1007/3-540-26669-0_1

Feist, G. J. (2006). The psychology of science and the origins of the scientific method. New Haven: Yale University Press.

Gergen, K. J. (1985). The social constructionist movement in modern psychology. American Psychologist, 40, 266-275. doi: 10.1037/0003-066X.40.3.266

Green, J., \& Thorogood, N. (2004). Qualitative methods for health research. London: Sage.

Hacking, I. (1995). The looping effect of human kinds. En D. Sperber, D. Premack \& A. J. Premack (Eds.), Causal cognition: a multidisciplinary debate (pp. 351-383 y pp. 384-394). Oxford, RU: Clarendon. doi: 10.1093/ acprof:oso/9780198524021.003.0012

Haely, K. C. (2008). Objectivity in the feminist philosophy of science. London: Continuum International.

Hanson, N. R. (1971). Patrones de descubrimiento. Madrid: Alianza.

Harding, S. (1999). After the neutrality ideal: Science, politics, and «strong objectivity». En E. C. Polifroni \& M. Welch (Eds.), Perspectives on philosophy of science in nursing: an historical and contemporary anthology (pp. 451-461). Filadelfia: Lippincott, Williams \& Wilkins.

Heisenberg, W. (1958). Physics and philosophy. The revolution in modern science. New York: Harper \& Brothers.

Hessen, B. (2009). The social and economic roots of Newton's Principia. En G. Freudenthal \& P. McLaughlin (Eds.), The social and economic roots of the scientific revolution (pp. 41-101). New York: Springer. doi: 10.1007/978-1-4020-9604-4_2
Hicks, S., \& Taylor, C. (2008). A complex terrain of words and deeds: Discourse, research, and social change. En P. Cox, T. Geisen \& R. Green (Eds.), Qualitative research and social change. European contexts (pp. 52-72). New York: Palgrave Macmillan. doi: 10.1057/ 9780230583962_4

Isambert, F. A. (1985). Un «programme fort» en sociologie de la science?. A propose de plusieurs ouvrages de sociologie de la science. Revue Française de Sociologie, 26(3), 485-508.

Kirk, R. (1999). Relativism and reality. A contemporary introduction. London: Routledge.

Kuhn, T. S. (1977). The essential tension. Selected studies in scientific tradition and change. Chicago: Chicago University Press.

Lakatos, I. (1978). Philosophical papers. Vol. 1. Cambridge, RU: Cambridge University Press.

Laudan, L. (1977). Progress and its problems. Berkeley, CA: University of California Press.

Longino, H. E. (1990). Science as social knowledge: Values and objectivity in scientific inquiry. Princeton: Princeton University Press.

MacKinnon, C. A. (1991). Toward a feminist theory of the state. Cambridge, MA: Harvard University Press.

McGrath, J. E., \& Johnson, B. A. (2003). Methodology makes meaning: How both qualitative and quantitative paradigms shape evidence and its interpretation. En P. M. Camic, J. E. Rhodes, \& L. Yardley, L. (Eds.) Qualitative research in psychology: Expanding perspectives in methodology and design, (pp. 31-48). Washington: American Psychological Association. doi: 10.1037/10595-003

Méndez, E. (2000). El desarrollo de la ciencia. Un enfoque epistemológico. Espacio Abierto, 9, 505-534.

Norris, C. (2003). Quantum theory and the flight from realism. Philosophical responses to quantum mechanics. New York: Routledge.

Ponterotto, J. G. (2005). Qualitative research in counseling psychology: a primer on research paradigms and philosophy of science. Journal of Counseling Psychology, 52, 126-136. doi: 10.1037/0022-0167.52. 2.126 
Potter, E. (2006). Feminism and philosophy of science. An introduction. London: Routledge.

Rapport, N. (2004). From the porter's point of view. In F. Rapport (Ed.), New qualitative methodologies in health and social care research (pp. 99-122). New York: Routledge.

Sceski, J. (2007). Popper, objectivity and the growth of knowledge. New York: Continuum.

Searle, J. R. (1993). Rationality and realism, what is at stake? Daedalus, 122(4), 55-83.
Sokal, A., \& Bricmont, J. (1999). Imposturas intelectuales. Barcelona: Paidós Ibérica.

Stanley, L. (Ed.) (2013). Feminist praxis. Research, theory and epistemology in feminist sociology. London: Routledge.

Vanderstoep, S. W., \& Johnston, D. D. (2009). Research methods for everyday life. Building qualitative and quantitative approaches. San Francisco: Jossey-Bass.

Willig, C. (2013). Introducing qualitative research in psychology. Adventures in theory and method ( $3^{\mathrm{a}}$. ed.). Maidenhead, RU: Open University Press.

\section{Roberto Bueno-Cuadra}

Universidad de San Martín de Porres

Universidad Nacional Federico Villarreal

Doctor en Psicología. Premio Nacional de Psicología otorgado por el Colegio de Psicólogos del Perú. Investigador calificado por el Consejo Nacional de Ciencia, Tecnología e Innovación Tecnológica.

Autor corresponsal: rbuenoc@usmp.pe; robuenoc@hotmail.com

Elizabeth Dany Araujo-Robles

Universidad Peruana Cayetano Heredia

Doctora en Psicología. Docente universitaria en los niveles de pre-grado y post-grado. Expositora en certámenes científicos internacionales y autora de publicaciones en revistas indexadas.

elizadany@hotmail.com 